\title{
Pourquoi la pisciculture tarde à se développer dans la ville de Bertoua (Est-Cameroun)?
}

\author{
Mohamadou MAIMOUNE ${ }^{1}$, Elvis Mouyakan A MOUMBOCK ${ }^{2}$, Emmanuel BAYOI $^{3}$, \\ $\mathrm{ASEDD}^{4}$ et Mbezele Junior Yannick NGABA ${ }^{5 *}$ \\ ${ }^{\text {I} I n s t i t u t ~ S u p e ́ r i e u r ~ d ' A g r o n o m i e ~(I S A) ~ d e ~ B e r t o u a, ~ U n i v e r s i t e ́ ~ d e ~ D s c h a n g, ~ B . P . ~} 222$ Dschang, \\ Cameroun. \\ ${ }^{2}$ Ministère de la Recherche Scientifique et de l'Innovation (MINRESI), Yaoundé, Cameroun. \\ ${ }^{3}$ Station de Vulgarisation Agricole d'Abong Mbang \\ ${ }^{4}$ Association des Experts pour le Développement Durable, \\ ${ }^{5}$ College of Natural Resources and Environment, Northwest A\&F University, Yangling 712100, Shaanxi, \\ China. \\ *Corresponding author; E-mail: ngabajunior@yahoo.fr, Tel: +86 18305996238,
}

\begin{tabular}{lll}
\hline Received: 04-07-2021 & Accepted: 22-11-2021 & Published: 31-12-2021 \\
\hline
\end{tabular}

\section{RESUME}

Le poisson est une source de protéine animale importante pour les populations et la plus consommée au Cameroun. Le développement de ce secteur est par conséquent un enjeu majeur pour la sécurité alimentaire. La présente étude menée dans la ville de Bertoua avait pour objectif de contribuer au développement du secteur aquacole au Cameroun en faisant un état des lieux. Les données ont été collectées à travers des entretiens faits sur 99 répondants et de la revue de littérature. Il ressort des analyses que $76 \%$ des acteurs sont des hommes âgés majoritairement entre 50-60 ans. Le Kanga (Heterotis niloticus) (25\%) et la carpe commune (Cyprinus Carpio) (26,19\%) sont les espèces les plus produites. Toutefois, malgré le potentiel hydrologique de la ville de Bertoua, on a observé un déclin du nombre de pisciculteurs et par conséquent du taux de production entre 2015 et 2018. D'autres facteurs par ailleurs constituent également un frein à l'essor de la pisciculture tels que la mauvaise gestion de l'étang (45,31\%), les contraintes institutionnelles $(34,37 \%)$ et la faible connaissance des techniques d'aménagement de l'étang (20,32\%). Afin de favoriser le développement de ce secteur et d'encourager les populations locales, des recommandations ont été faites. Entre autres, le recyclage et l'appropriation de nouvelles techniques piscicoles par les populations; l'amélioration et la facilitation du processus d'acquisition des licences, le développement de stratégies visant à faciliter l'accès aux intrants nécessaires à la production piscicole: l'appui technique des aquaculteurs lors de la construction des infrastructures et dans l'acquisition des terres et des financements. La mise en place d'une politique aquacole nationale en utilisant une approche participative est nécessaire pour rendre effective le développement du secteur piscicole dans la région de l'Est.

(C) 2021 International Formulae Group. All rights reserved.

Mots clés : Poisson, aquaculture, produits halieutiques, Bertoua. 


\title{
Why is fish farming slow to develop in the city of Bertoua (East Cameroon)?
}

\begin{abstract}
Fish is an important source of animal protein for the populations and the most consumed in Cameroon. The development of this sector is, therefore, a major issue for food security. The objective of this study carried out in Bertoua was to contribute to the development of the aquaculture sector in Cameroon by assessing the current state. Data were obtained through interviews with 99 respondents and a literature review. The analysis revealed that $76 \%$ of the stakeholders were men, mostly between 50 and 60. Kanga (Heterotis niloticus) (25\%) and common carp (Cyprinus Carpio) (26.19\%) are the most produced species. However, despite the hydrological potential of Bertoua City, there has been a decline in the number of fish farmers and consequently in the production rate between 2015 and 2018. Other factors, also constitute a hindrance to the development of fish farming, such as poor pond management $(45.31 \%)$, institutional constraints $(34.37 \%)$, and insufficient knowledge of pond management techniques $(20.32 \%)$. To promote the development of this sector and to encourage local populations, made recommendations. Among others, the recycling and appropriation of new fish farming techniques by the people; the improvement and facilitation of the process of acquiring licenses, the development of strategies to facilitate access to the inputs necessary for fish farming production; the technical support of fish farmers during the construction of infrastructures and in the acquisition of land and financing. Establishing a national aquaculture policy using a participatory approach is necessary to make the development of the fish farming sector in the Eastern region effective.
\end{abstract}

(C) 2021 International Formulae Group. All rights reserved.

Keywords: Fish, aquaculture, fishery products, Bertoua.

\section{INTRODUCTION}

Le poisson constitue la protéine animale la plus consommée au Cameroun pour plus de 11 millions d'habitants. Il représente environ $44 \%$ des protéines animales et $7,6 \%$ des apports en protéines totales (Fongang, 2008). Sa consommation varie entre 10 à $45 \mathrm{~kg}$ par habitant et il est très prisé par les couches les plus défavorisées (Tambi, 2001 ; FAO, 2016). Ce rôle pourrait devenir plus important dans le futur, car la demande est sans cesse croissante. Au regard de l'importance que joue cette ressource pour la sécurité alimentaire des populations, le Cameroun importe près 200000 tonnes de poissons par an et dépense environ 170 milliards de FCFA/an (Ndoumbe, 2019). Selon les projections, cette dépendance aux importations va s'accentuer dans les années à venir, probablement à cause de la constance de la demande et du recul de la production halieutique locale (Teleu et Ngatchou, 2006 ;
Kenfack et al., 2019). En effet, l'achat de poissons est passé de 181678 tonnes de poissons surgelés en 2017 à 292675 tonnes en 2018, dont 50,6\%, sur le marché africain (FAO, 2016). Par ailleurs, malgré la hausse de la production halieutique et aquacole, la production nationale reste faible. La demande nationale a été estimée à environ 400000 tonnes par an au Cameroun et le déficit en production du poisson à près de 250 000 tonnes (INS, 2015). Or, les estimations des productions halieutiques au Cameroun en 2019 sont d'environ 289764 tonnes, dont 271317 tonnes de poissons pour la pêche artisanale, 11370 tonnes de poissons pour la pêche industrielle et 7077 tonnes de poissons pour l'aquaculture (Ecofin, 2019). Il est dès lors évident que les importations et la production nationale n'arrivent à satisfaire les besoins en ressources halieutiques de la population. 
La ville de Bertoua joue un rôle crucial dans l'approvisionnement en ressource halieutique de cette région (Baleng et al., 2020 ; Moumbock et al., 2020). De plus, l'arrivée massive des réfugiés ayant fui la crise en République Centrafricaine (RCA) a aggravé le besoin de cette ressource et augmenté les risques d'insécurité alimentaire dans la région. Fort de ce constat, les populations de la ville de Bertoua en particulier ont compris que l'aquaculture représente un levier crucial qui peut combler ce déficit et se sont depuis plusieurs années impliquées dans la production aquacole. C'est ainsi que cette activité est devenue non seulement une source de revenus pour ces producteurs locaux, mais elle peut également permettre de réduire le taux d'importations (FAO, 2007). Toutefois, les potentialités de cet élevage restent mal connues notamment en ce qui concerne les freins qui empêchent l'essor de cette activité dans la ville de Bertoua. Pourtant ces informations sont nécessaires, voire incontournables pour mieux définir et orienter les stratégies de gestion à envisager. Cette étude vise à fournir des informations sur l'élevage piscicole dans la ville de Bertoua, identifier les contraintes et opportunités de l'aquaculture dans cette zone et proposer des solutions visant à promouvoir l'activité agricole dans la ville de Bertoua.

\section{MATERIELS ET METHODES}

\section{Localisation et présentation de la zone d'étude}

Avec une superficie actuelle est estimée à $100 \mathrm{~km}^{2}$, la ville de Bertoua est située dans le Lom-et-Djérem dans la province de l'Est au Cameroun à $350 \mathrm{~km}$ de Yaoundé. Elle se localise entre le $4^{\circ} 57^{\prime}$ de latitude Nord et $13^{\circ} 66^{\prime}$ de Longitude Est
(Figure 1). C'est la capitale de la plus grande région forestière du Cameroun ce qui fait d'elle le principal pôle de développement de la région. Bertoua est drainée du nord au sud par le cours d'eau dénommé Djadombe et son altitude varie entre $400-900 \mathrm{~m}$. Les précipitations oscillent entre 1500 à $2000 \mathrm{~mm}$ par an et les températures varient entre $23^{\circ} \mathrm{C}$ et $25^{\circ} \mathrm{C}$. Il a dominé par un climat de type subtropical à trois saisons (une grande saison sèche qui va de décembre à mi-mars; une petite saison de pluies de mi-mars à mi-mai, une grande saison de pluies de mi-septembre à novembre) (MINEPAT, 2010).

\section{Collecte des données}

La collecte des données a eu lieu du 1er avril 2020 au 30 juin 2020 dans la ville de Bertoua. Les données ont été obtenues auprès des différents services publics Ministère De L'élevage Des Pêches Et Industries Animales (MINEPIA) et les aquaculteurs. Les questionnaires semi-structurés et des observations ont été utilisés pour les entretiens avec les responsables. La liste des acteurs a été obtenue auprès du MINEPIA ce qui nous a permis d'identifier et localiser les aquaculteurs et leurs sites. Au total, trenteneuf (39) aquaculteurs ont accepté participer à cette étude. Les informations recherchées étaient: les caractéristiques socioéconomiques des répondants, leurs activités; les difficultés qu'ils rencontrent et les mesures qu'ils entreprennent pour y faire face; leur perception par rapport à l'activité et leurs attentes vis-à-vis du gouvernement.

\section{Analyse des données}

Les données collectées ont été encodées et triées classées, à l'aide du logiciel Microsoft Excel 2016 et l'analyse des données via le logicien OriginePro version 8.5. 


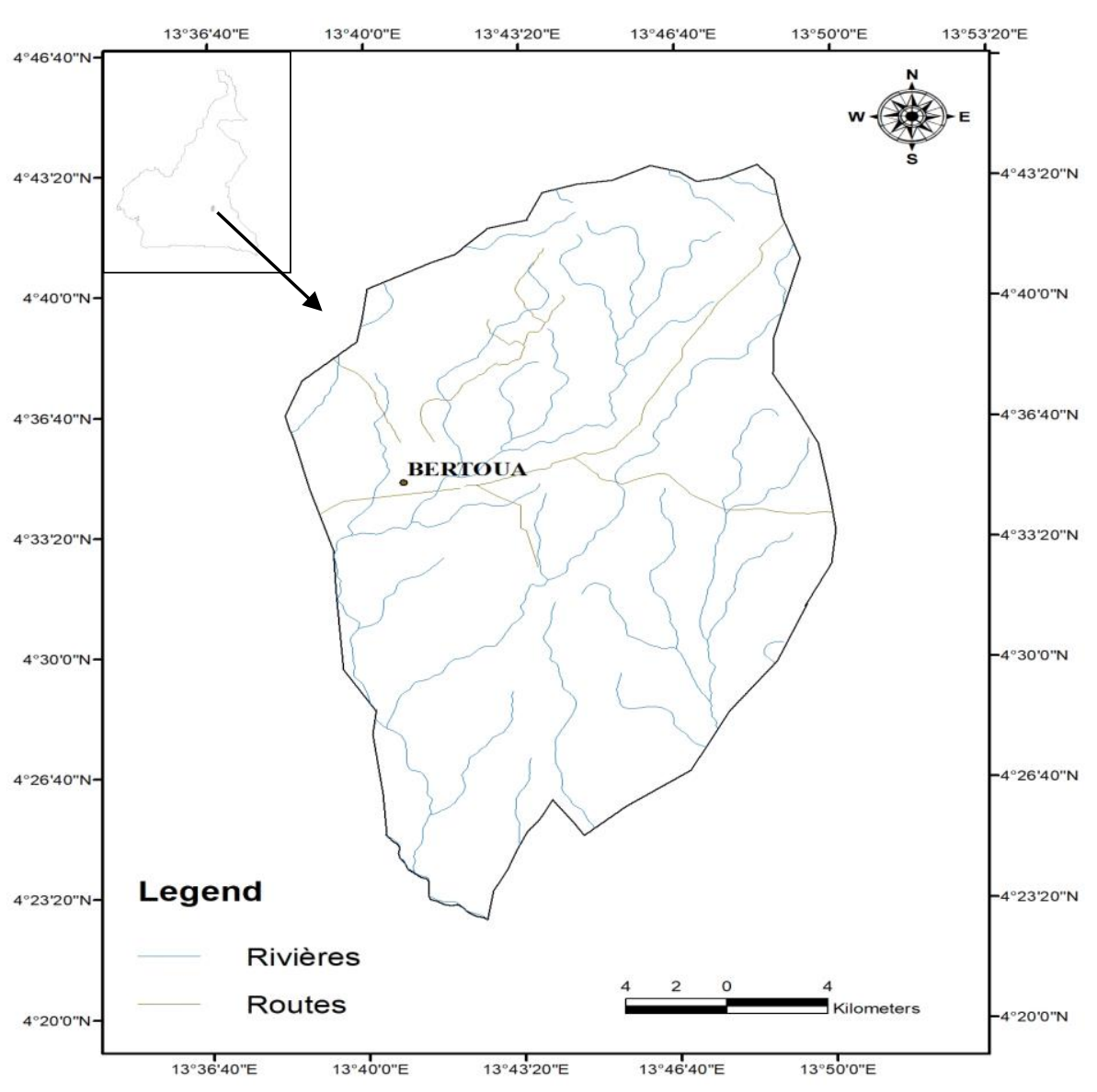

Figure 1: Localisation de la ville de Bertoua.

\section{RESULTATS}

\section{Caractérisation socio-économique des aquaculteurs}

évidence

Cette étude a permis de mettre en sociotechniques de l'aquaculture dans la ville de Bertoua. Les hommes pratiquent majoritairement cette activité. Cette tendance a été observée dans plusieurs études antérieures (Hirigoyen et al., 1997). Elle peut s'expliquer par le statut de la femme dans la zone. Il ressort du Tableau 1 que cette activité est plus pratiquée par les hommes, généralement des personnes âgées entre 50 et 60 ans. Comme ont également indiqué
Pouomogne et Pemsl (2008). Cela peut également se traduire par le fait que l'aquaculture est une activité qui prend du temps. Les personnes âgées de plus de 70 ans sont des aquaculteurs inactifs qui apportent généralement leur expérience à leur proche. La majorité des répondants sont au moins fait le primaire $(50 \%)$.

Les jeunes ( $<30$ ans) évitent cette activité parce qu'ils trouvent qu'elle ne rapporte pas assez de revenus comparés aux autres activités et prend du temps. Ils préfèrent par conséquent se tourner vers des activités à revenu immédiat telles que la pêche, le petit commerce ou travailler pour les 
sociétés qui s'installent dans la région. Le taux de scolarisation élevé des répondants $(93,3 \%)$ des acteurs prouve qu'ils exercent cette activité volontairement et non par contrainte sociale. L'activité principale exercée par les répondants était l'agriculture $(36,66 \%)$ suivie de ceux qui occupent un poste à la fonction publique $(23,33 \%)$. Les aquaculteurs représentent $3,3 \%$ des répondants. Ces résultats démontrent que l'aquaculture est considérée comme une activité secondaire dans la ville de Bertoua à caractère familial et ne se pratiquant pas le plus souvent à l'échelle industrielle.

D'autres études ont reporté des résultats similaires. Tiogué et al. (2020), dans la région du Centre, a reporté par exemple que cette activité était majoritairement pratiquée par les agriculteurs $(62,5 \%)$ et que la pisciculture était faiblement représentée (7,5\%). Les autres activités $(23,31 \%)$ incluses vétérinaires, le commerce, électricien, le génie civil. L'aquaculture dans la ville de Bertoua est majoritairement pratiquée par les Baya $(37,93 \%)$ suivi des bamilékés (17.24\%) et des Kako $(13,76 \%)$. Cette dominance peut s'expliquer par l'accessibilité aux terres. Les répondants ont pour activités principales l'agriculture $(30 \%)$ ou travaille pour le gouvernement $(23,33 \%)$ ce qui nous pousse à croire qu'ils considèrent ce secteur comme une source d'investissement.

\section{Caractérisation des élevages}

Le Tableau 2 nous montre que le Kanga (Heterotis niloticus) est l'espèce la plus produite $(26,19 \%)$ suivie de la carpe commune (Cyprinus carpio) $(25 \%)$ et du Tilapia (Oreochromis niloticus) $(20,23 \%)$. Le Kanga est plus produit dans la ville à cause de ces saveurs gustatives très appréciées par la population. Malheureusement, seulement 07 espèces sont produites dans la zone probablement à cause de la parfaite maitrise de production de ces espèces. L'Hemichromis joue un rôle de contrôle de la faune aquatique. Cette faible diversité d'espèce de poisson s'explique par le fait que l'approvisionnement en alevins dans la zone est très difficile à cause du manque/l'insuffisance d'écloserie.

Trois types d'étangs sont rencontrés dans la ville : les étangs de barrage, les étangs de dérivation et les étangs de nappe phréatique (zone de marécage). Il est à noter que les étangs de barrage sont les plus utilisés $(83,33 \%)$ probablement à cause du faible coût de construction (Photo 1). Le système de production exercé dans la localité est de type extensif. L'alimentation des poissons n'est pas accentuée et dans certains étangs, les poissons sont nourris naturellement. En général, $34.48 \%$ des aquaculteurs envisagent effectuer ce métier sur 10 ans et $27,58 \%$ sur 20 ans. Le poisson est généralement consommé sous des formes très diverses (frais, séchés, fumés ou fermentés), l'excédent est vendu ou donné aux proches.

\section{Évolution de l'aquaculture dans la ville de Bertoua}

Au fil des années, le secteur piscicole a connu beaucoup de changements qu'il s'agisse du nombre d'acteurs, d'étangs actifs ou de la production. La Figure 2 renseigne sur l'évolution de cette activité sur 05 ans précisément entre 2015 et 2019 ces cinq dernières années. Il ressort que le taux d'aquaculteurs et d'étangs ont diminué de $5,95 \%$ et $9,2 \%$ respectivement entre 2015 et 2019. Tandis que le taux de production de poissons a diminué entre 2015 et 2018 de 20,38\% et a augmenté entre 2018 et 2019 de $355.66 \%$. Ces résultats démontrent que l'efficacité des aquaculteurs de production a augmenté durant cette période. Contrairement à l'augmentation des acteurs de cette activité dans la ville de Yaoundé (MINEPIA, 2015; Tomedi, 2015), la population de la ville de Bertoua semble de plus en plus désintéressée probablement à cause des faibles campagnes de vulgarisation et promotion de cette activité. Malheureusement, les populations payent le prix fort avec l'augmentation des prix des poissons sur le marché. 
Tableau 1: Caractéristiques sociodémographiques des répondants.

\begin{tabular}{|c|c|c|}
\hline Variables & Caractéristiques & Fréquences $(\%)$ \\
\hline \multirow{2}{*}{ Sexe } & Homme & 76,6 \\
\hline & Femme & 24,4 \\
\hline \multirow{6}{*}{ Âge (ans) } & $<30$ & I \\
\hline & $30-40$ & 17,85 \\
\hline & $40-50$ & 17,85 \\
\hline & $50-60$ & 32,15 \\
\hline & $60-70$ & 28,17 \\
\hline & $>70$ & 3,58 \\
\hline \multirow{5}{*}{ Situation matrimoniale } & Célibataire & 10 \\
\hline & Marié & 66,66 \\
\hline & Veuf & 10 \\
\hline & Divorcé & 3,33 \\
\hline & Inconnu & 10 \\
\hline \multirow{6}{*}{ Nombre d'enfants } & $1-5$ & 36,66 \\
\hline & $5-10$ & 36,66 \\
\hline & $10-15$ & 13,33 \\
\hline & Nombre inconnu & 13,33 \\
\hline & Primaire & 16,66 \\
\hline & Secondaire & 50 \\
\hline \multirow{3}{*}{ Niveau de scolarisation } & Universitaire & 13,33 \\
\hline & Non précisée & 13,33 \\
\hline & Non scolarisé & 6,68 \\
\hline \multirow{6}{*}{ Principale activité } & Agriculture & 36,67 \\
\hline & Fonction publique & 23,34 \\
\hline & Hôtellerie & 6,67 \\
\hline & Maçonnerie & 6,67 \\
\hline & Aquaculture & 3,34 \\
\hline & Autres & 23.31 \\
\hline \multirow{10}{*}{ Groupe ethnique } & Baya & 37,93 \\
\hline & Bamilékés & 17,24 \\
\hline & Kako & 13,76 \\
\hline & Maka & 6,89 \\
\hline & Paul & 6,89 \\
\hline & Wobekolo & 3,44 \\
\hline & Bakom & 3,44 \\
\hline & Mbamvélé & 3,44 \\
\hline & Bulu & 3,44 \\
\hline & Bobolis & 3,44 \\
\hline \multirow{2}{*}{ Formation } & Professionnel & 34.17 \\
\hline & Pas de formation & 65.83 \\
\hline \multirow{5}{*}{$\begin{array}{l}\text { Source de financement } \\
\text { de l'exploitation }\end{array}$} & Agriculture & 30 \\
\hline & Commerce & 3,33 \\
\hline & Pisciculture/Pêche & 3,33 \\
\hline & Salaires & 23,33 \\
\hline & Autres & 39,99 \\
\hline
\end{tabular}


Tableau 2: Caractérisation de l'aquaculture dans la ville de Bertoua.

\begin{tabular}{lll}
\hline \multirow{2}{*}{ Variables } & $\begin{array}{l}\text { Noms communs et noms } \\
\text { scientifiques }\end{array}$ & Fréquence (\%) \\
\hline & Kanga (Heterotis niloticus) & 26,19 \\
& Carpe commune (Cyprinus carpio) & 25 \\
& Tilapia (Oreochromis niloticus) & 20,23 \\
Espèces produites & Silure (Clarias gariepinus) & 16,66 \\
& Poisson-vipère (Chauliodus) & 7,14 \\
& Hemichromis (Hemichromi sfasciatus) & 3,57 \\
& Carpe noire (Mylopharyng donpiceus) & 1,19 \\
\hline \multirow{4}{*}{ Trois types d'étangs } & Les étangs de barrage & 83,33 \\
& Les étangs de dérivation et & 6,66 \\
& Les étangs de nappe phréatique & 10 \\
\hline \multirow{3}{*}{ Aliments utilisés lors de } & Ménagers & $/$ \\
l'élevage & Agricoles & 30 \\
& Mixtes & 13,33 \\
& Naturellement & 56,66 \\
\hline \multirow{2}{*}{ Destination de la récolte } & Consommation & 3,33 \\
Nombre d'enfants & Commerce & 33,33 \\
& Commerce et consommation & 3,33 \\
& Commerce et don & 3,33 \\
& Commerce, consommation et don & 6,66 \\
\hline
\end{tabular}

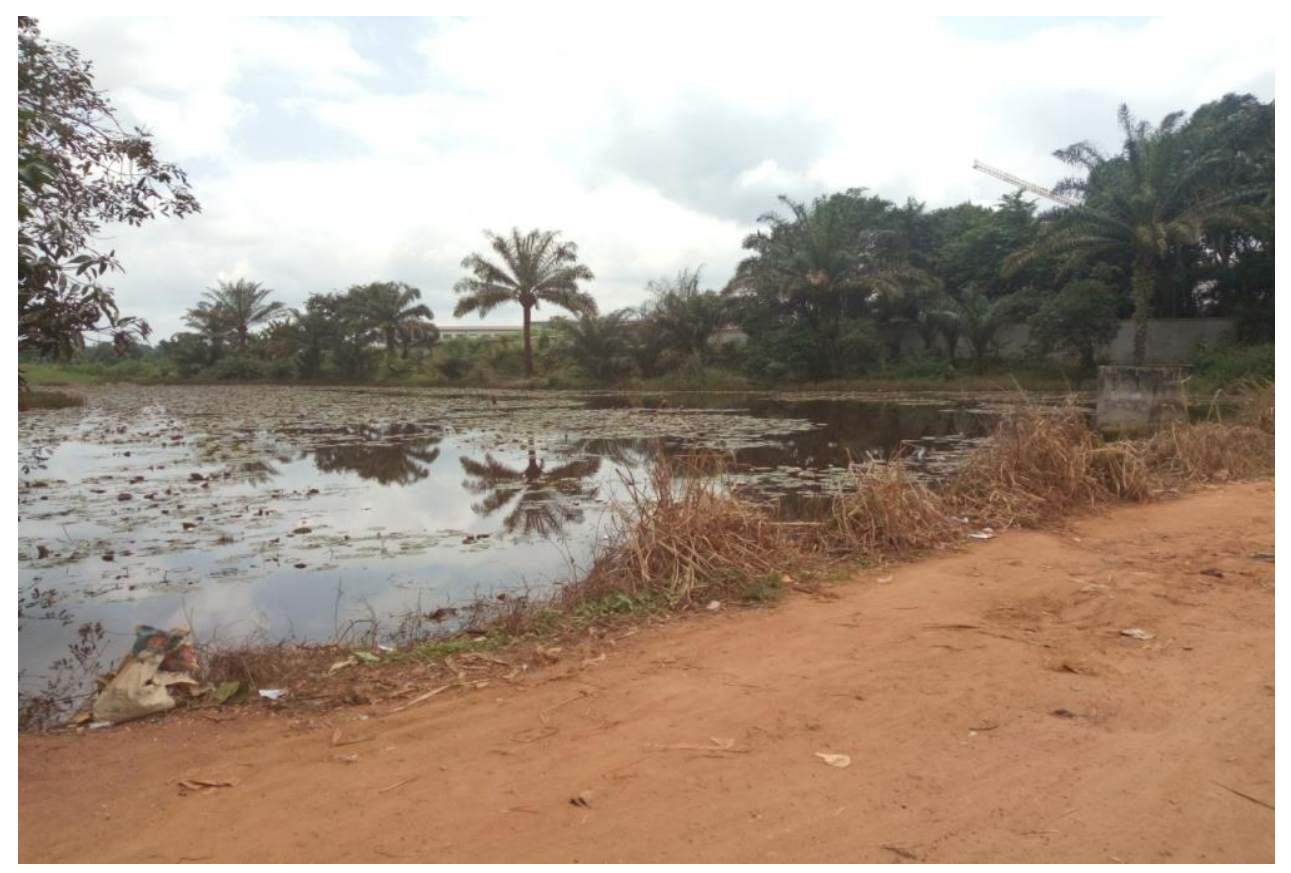

Photo 1: Étang de barrage situé à Nkolbikon-Ndjamena (Est-Cameroun, Bertoua). 


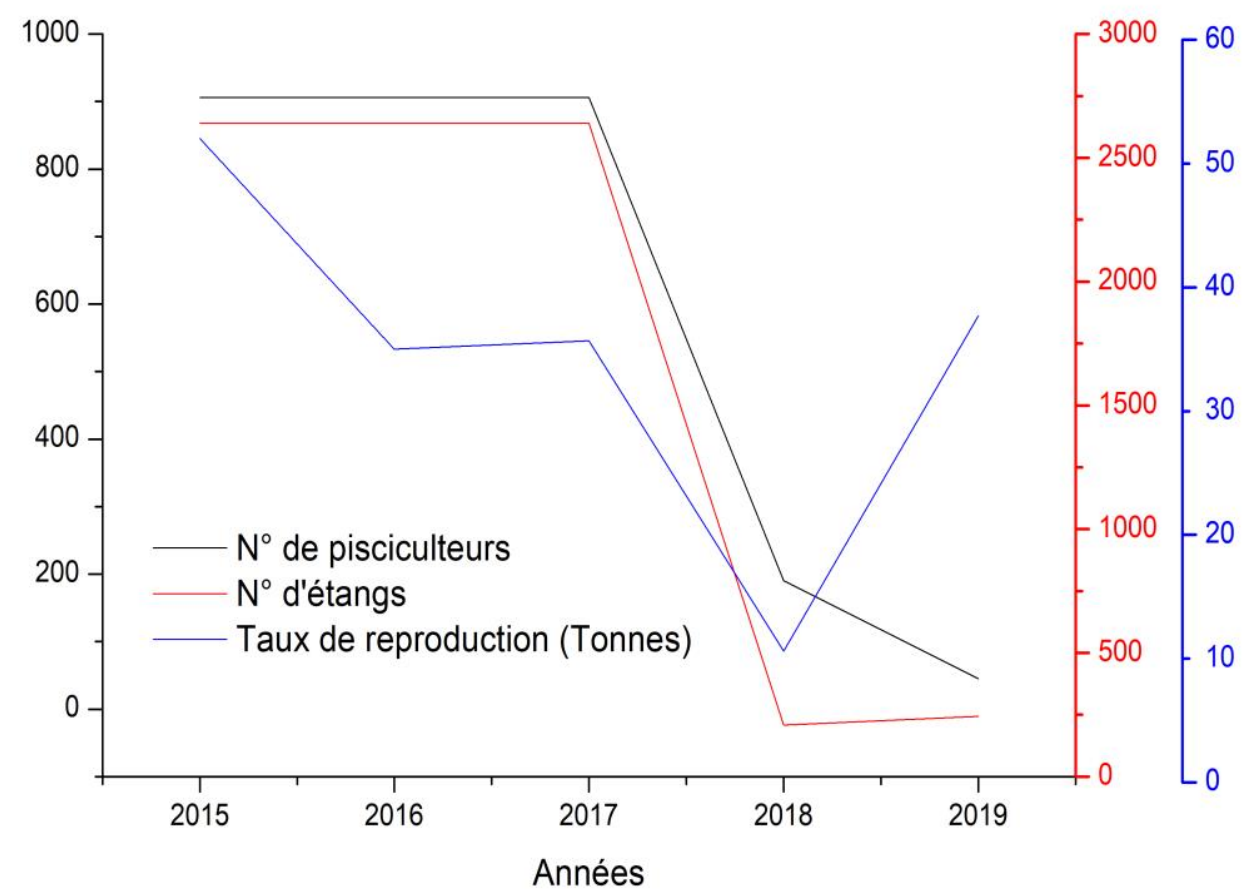

Figure 2: Évolution de l'activité aquacole entre 2015 et 2019 de la ville de Bertoua.

\section{DISCUSSION}

\section{Freins au développement de l'aquaculture}

Les acteurs de filière aquacole dans la ville de Bertoua rencontrent plusieurs difficultés en fonction de la typologie des contraintes comme l'indique le Tableau 3. Selon les répondants, elles sont de trois ordres : la gestion de l'étang (45,31\%), les contraintes institutionnelles (34,37\%), l'aménagement de l'étang $(20,32 \%)$ et sont les difficultés les plus rencontrées. Le non-accès aux crédits limite l'amélioration des pratiques et par conséquent du rendement de production. Toutefois, l'insuffisance des ressources financières reste la difficulté la plus rencontrée par les aquaculteurs. En effet, l'achat d'aliments représente 50 à $70 \%$ des facteurs de production (Oswald et Mikolasek, 2016). De plus, le prix des ressources importé (poissons importés «congelés») et souvent moins élevé que ceux produits localement ce qui décourage souvent les populations. La rentabilité de cette activité reste mitigée, car elle est très influencée par plusieurs facteurs : les charges sur l'aliment, un manque de visibilité des objectifs fixés; la fluctuation des coûts des matières premières nécessaires à l'élevage ou l'insuffisance sur les aspects techniques et matériels.

La mauvaise qualité des routes rend les fermes difficiles d'accès. Ce qui a des effets sur l'approvisionnement en intrants (fourniture d'alevins, nourriture, matières premières), la fréquence du suivi des élevages tant pour les aquaculteurs que par les agents de vulgarisation (Kenfack et al., 2019 ; Sarr et al., 2015). Bien que plusieurs points positifs aient été relevés en ce qui concerne le rôle joué par le MINEPIA notamment: la décentralisation des services administratifs; 
l'encadrement des opérateurs économiques ; la mise en place d'un système de collecte des données ou la bonne diffusion des informations sur les nouvelles technologies plusieurs facteurs l'empêchent d'accomplir pleinement ces fonctions (Tableau 3). D'après Kenfack et al. (2019), le secteur aquacole est totalement sinistré, pourtant le pays dispose de tous les éléments nécessaires pour développer une aquaculture durable et rentable à savoir une politique nationale de la pêche et de l'aquaculture. En général, les problèmes institutionnels sont issus des prises de décision basées sur des informations qui ne cadrent pas avec les réalités de terrain et l'utilisation de lois obsolètes (N'dri et al., 2021).

\section{Opportunités et recommandations}

En dépit du fait que plusieurs répondants affirment avoir reçu une formation $(36,17 \%)$, d'être assistés par le MINEPIA $(34,04 \%)$ et par d'autres partenaires $(29,78 \%)$ beaucoup d'opportunités restent à saisir (inexistantes et/ou potentielles) et à encourager (existants avec nécessité d'être implémenté) comme l'indique le Tableau 4. On peut citer entre autres l'augmentation des acteurs et espaces consacrés à cette activité. Afin d'atteindre cette objectif, les institutions en charge de sa gestion mettent sur pied un cadre favorable pour faciliter l'accès à cette activité. La diversification des acteurs/investisseurs pourra ainsi permettre l'utilisation et la vulgarisation de nouvelles techniques telle que l'élevage en cage de poissons. Elle peut également permettre l'émergence de nouvelles entreprises dans le secteur aquacole créer de nouveaux emplois et contribuer à améliorer leur sécurité alimentaire. Le potentiel d'intégration de la recherche appliquée à l'aquaculture est largement reconnu comme un moyen primordial pour l'amélioration du rendement. Particulièrement à travers l'amélioration d'utilisation des intrants, la diversification de la production et les possibilités économiques en vue de permettre aux petits producteurs de maintenir et de renforcer leurs moyens de subsistance (Brummett et Jamu, 2011). Les facteurs climatiques sont également un atout dans la zone. En effet, la température moyenne dans cette localité est de $24^{\circ} \mathrm{C}$ ce qui permet l'élevage de plusieurs espèces de poissons tropicales qui se développent aisément dans ces conditions. Le réseau hydrographique représente un facteur primordial de réussite de la pisciculture, car il facilite l'élevage des poissons en étangs sur des plans d'eau naturels tels que les cages flottantes.

Plusieurs études ont relevé le fait que les pisciculteurs ne sont pas toujours conscients de l'existence de la règle et ne comprennent pas cette dernière (Kenfack et al., 2020). Ce constat de leur méconnaissance de la réglementation ou à leur incapacité à la respecter implique que la plupart des pisciculteurs dans la ville de Bertoua exercent dans l'informel. En outre, qu'il y a une très faible sensibilisation sur la réglementation en vigueur auprès de ses acteurs. Il est primordial que les populations se sentent davantage concernées par le respect des textes afin de ne pas se retrouver dans l'infraction. Ce contexte favorise le gré à gré, par conséquent la corruption et constitue de ce fait un obstacle significatif au développement de l'aquacole dans la ville de Bertoua. Le MINEPIA doit mettre en place des modalités de collecte et de conservation des produits de l'aquaculture et des moyens pour encourager les partenariats entre les aquaculteurs et les acteurs impliqués dans la commercialisation du poisson. Cette mesure va permettre aux pisciculteurs d'écouler facilement leurs productions lors des marchés périodiques ou dans les sites de vente.

En ce qui concerne la promotion de l'aquaculture, les actions menées doivent porter sur plusieurs axes à savoir : l'encouragement des populations à l'activité par des campagnes de sensibilisation; l'amélioration de la production des écloseries et des fermes aquacoles par la maîtrise de l'élevage larvaire et de la gestion technicoéconomique; et la vulgarisation des techniques d'élevage de poissons en cage, 
avec l'appui des partenaires (Yoboue et al., 2018). Par ailleurs, l'octroi des licences est un axe primordial pour l'évolution de ce secteur. Elles permettent d'établir la transparence des acteurs et d'améliorer l'acceptabilité sociale de l'activité aquacole (Kenfack et al., 2019). Toutefois, l'installation d'un centre sanitaire spécialisé doit suivre avec l'implémentation de toutes ses mesures. La libéralisation de ce secteur comme souhaité doit être contrôlée et suivie notamment sur la qualité de la ressource mise à la disponibilité de la population afin d'éviter des cas d'intoxication et de perte en vie humaine. Le Centre de Recherche et d'éducation pour le Développement (CREPD) avait par exemple rapporté que le poisson d'Ayos et de Youpwe impropre à la consommation à cause de leur forte teneur en mercure (Ebosse, 2014). Le contexte halieutique peut changer si certaines mesures sont mises en place. Il est difficile de comprendre pourquoi une seule compagnie (CONGELCAM) contrôle plus de $80 \%$ du marché du poisson au Cameroun (Ndoumbe, 2019).

Tableau 3: Problèmes rencontrés par les aquaculteurs dans la ville de Bertoua.

\begin{tabular}{ll}
\hline Typologie des contraintes & Problèmes rencontrés \\
\hline \multirow{3}{*}{ Aménagement des étangs } & Manque/insuffisance de ressources financières \\
& Difficulté d'accès aux terres \\
& Indisponibilité d'une main-d'œuvre qualifiée \\
& Difficulté d'accès au crédit \\
& Coûts élevés des études géologiques \\
\hline & Equipements obsolètes et non adaptés \\
& Manque/insuffisance de ressources financières \\
& Rupture de digue \\
& Faible rendement \\
& Indisponibilité d'une main-d'œuvre qualifiée \\
& La prolifération des plantes aquatiques \\
& Vol/braconnage (prédation des poissons qui peut être humaine ou animale) \\
& Problème de crue \\
& Absence de route de qualité \\
& Conflits liés à l'utilisation des terres \\
& Pollution des cours d'eau \\
\hline Gestion des étangs & Manque/insuffisance d'appui technique \\
& Manque/insuffisance d'alevins et prix élevé \\
& Manque de moyens logistiques \\
& Insuffisance de coordination et d'encadrement par les \\
agents de vulgarisation & Manque de personnel de terrain \\
& Insuffisance de personnel qualifié dans le domaine de la valorisation et conservation \\
des produits de la pêche & Taxes élevées sur les bénéfices des sociétés \\
& Fluctuation des prix des alevins \\
Inexistence d'un tissu industriel adéquat pour la transformation des produits \\
d'élevage \\
Méconnaissance de la procédure d'autorisation de création de fermes piscicoles \\
Insuffisance de site de recherche \\
Concurrence inégale avec les produits importés (prix) \\
\hline Institutionnelles & \\
\hline
\end{tabular}


Tableau 4: Opportunités et recommandation pour l'essor de la pisciculture dans la ville de Bertoua.

\section{Opportunités liées à l'activité piscicole}

\begin{tabular}{ll}
\hline & Demande élevée \\
& Diversification des acteurs ou opérateurs économiques (investisseurs) \\
Directes & Partenariat entre chercheurs et acteurs de terrain \\
& Utilisation de nouvelle technique (pisciculture en cage) \\
& Promotion de l'aquaculture comme secteur pourvoyeur d'emploi \\
& Diversification des espèces élevées \\
\hline & Amélioration des conditions socioéconomiques de la population \\
& L'existence d'un réseau hydrographique dense \\
& Température moyenne favorable à l'élevage des poissons \\
& Création d'un domaine d'exploitation proche du client et fonction du produit \\
& qu'il consomme (circuit de commercialisation) \\
& Assurer un accompagnement, un encadrement technique, un suivi et un \\
& ravitaillement en matériel adéquat pour les pisciculteurs \\
\hline
\end{tabular}

\section{Recommandations aux différents acteurs}

\section{Respect des textes en vigueur}

Aux acteurs Recyclage et appropriation de nouvelles techniques piscicoles

Impliquer davantage les femmes dans les projets aquacoles

Assurer la disponibilité des espèces piscicoles de fortes performances
génétiques
Améliorer et faciliter le processus d'acquisition des licences
Effectuer des recherches sur les techniques de production et conservation
Assister les aquaculteurs lors de la construction des infrastructures et faciliter
leur accès aux sites
Faciliter l'accès aux intrants nécessaires à la production piscicole
Accompagner les acteurs dans l'acquisition des financements et des terres
Accompagner techniquement par des formations sur l'utilisation des
nouvelles technologiques de pointe et des techniques améliorées




\section{Conclusion}

$\mathrm{Au}$ ter de cette étude ou il était question de faire une présentation du secteur aquacole dans la ville de Bertoua, il ressort que malgré les efforts consentis, le développement du secteur n'est pas assez promu et que cette activité n'est pas encore perçue comme un secteur rentable ou d'avenir par la population. Par ailleurs, les espèces les plus prisées de la production aquacole dans la ville de Bertoua sont les tilapias, le poissonchat nord-africain et les carpes communes. Les principaux défis rencontrés par les exploitants piscicoles sont le braconnage, les prédateurs et la pénurie de nourriture, le prix élevé des aliments commerciaux pour poissons, et les fonds limités du gouvernement pour la recherche et la vulgarisation du secteur.

\section{CONFLITS D'INTÉRÊTS} d'intérêts.

Les auteurs ne déclarent aucun conflit

\section{CONTRIBUTIONS DES AUTEURS}

EMAM et MN ont fait la conception de l'étude. MM a collecté les données de terrain. EMAM, BE et ASEDD ont préparé les résultats et rédigé le premier draft. $\mathrm{MN}$, EMAM et $\mathrm{BE}$ ont révisé et préparé le document final.

\section{REMERCIEMENTS}

Les auteurs remercient tout le personnel et corps enseignant du DEPFOR, pour leurs conseils, appuis techniques, encouragements et disponibilité lors de la réalisation de cette étude.

\section{REFERENCE}

Baleng RDN, Moumbock EMA, Ngaba MJY. 2020. Contribution à l'élaboration d'un plan simple de gestion d'une forêt communautaire: Cas de l'inventaire d'aménagement de la forêt d'Alati (SudCameroun). J. Cameroon Acad. Sci., 16(1): 53-69. DOI: 10.4314/jcas.v16i1.5.

Brummett RE, Jamu DM. 2011. From researcher to farmer: Partnerships in integrated aquaculture-Agriculture systems in Malawi and Cameroon. Int. J. Agric Sustain., 9(1): 282-289. DOI: 10.3763/ijas.2010.0570.

Ebosse. $2014 . \quad$ Site Internet. https://fr.allafrica.com/stories/20141103 1780.html.

Ecofin. 2019. Site Internet. https://www.scribbr.fr/citation-dessources/citer-un-siteinternet/\#: :text=Format\%20MLA,Nom\%20de\%20l'auteur\%2C\%20Pr\%C3 $\%$ A9nom.,Consult\%C3\%A9\%20le\%20 Date $\% 20$

FAO. 2006. The state of world highly migratory, straddling, and other high seas fishery resources and associated species, by J.-J. Maguire, M. Sissenwine, J. Csirke, r. Grainger and S. Garcia. FAO Fisheries technical Paper No. 495. rome.

Hirigoyen JP, Manjeli Y, Moucharou GC. 1997. Caractéristiques de la pisciculture dans la zone forestière du centre Cameroun. Tropicultura, 15(4): 180-185.

Institut national de statistique (INS). 2015. Chapitre 15: Elevage et Pêche. In Annuaire Statistique du Cameroun. INS, Edition 2015: 257-268.

Kenfack JA, Ducarme C, Micha J. 2019. La pisciculture au Cameroun: bilan et perspectives. International Journal of Biological and Chemical Sciences, 13(2): $\quad 1140-1161 . \quad$ DOI: 10.4314/ijbcs.v13i2.44.

Kenfack, JA, Tchawa P, Micha JC. 2020. Tendance au non-respect de la réglementation sur l'aquaculture au Cameroun. Geo-Eco-Trop., 44(2): 303312. DOI: $10.1051 /$ cagri/2019027.

MINEPAT, 2010. Cadre de gestion environnementale et sociale du projet compétitivité des filières de croissance (PCFC).

MINEPIA, 2015. Rapport d'activités : Le Cameroun multiplie des initiatives dans l'aquaculture pour booster sa production de poissons - Investir au Cameroun, p. 25.

Moumbock EMA, Ngaba MJY, Tsi EA. 2020. Gestion durable des Hautes Valeurs de 
Conservation de types 5 et 6 dans les UFA gérées par la société forestière Pallisco : Identification, cartographie et enjeux pour la conservation. April 2020. Edition : Volume 1. Publisher : Université de Dschang. Editor : Faculté d'Agronomie et des Sciences (FASA).

N'dri KM, Brou GKG, Yao KP and Diomande D. 2021. Caractérisation de la pisciculture dans la région du Poro (Nord de la Côte d'Ivoire). Int. J. Biol. Chem. Sci., 15(3): 976-986. DOI: 10.4314/ijbcs.v15i3.11.

Ndoumbe. 2019. Site Internet. https://actucameroun.com/2019/01/21/ca meroun-dangote-veut-investir-danslimportation-du-poisson.

Oswald M, Mikolasek O. 2016. Le secteur piscicole en Afrique subsaharienne : des outils de financement adaptés aux enjeux? Techniques Financieres et Developpement, 124(3): 81. DOI: 10.3917/tfd.124.0081.

Pouomogne V, Pemsl DE. 2008. Recommendation domains for pond aquaculture. Country Case study: Development and status of freshwater aquaculture in Cameroon-Worldfish Center Studies and Reviews $\mathrm{N}^{\circ} 1871$, The WorldFish Center (Ed.), Penang (Malaysia), p 60.

Sarr SM, Thiam A, Sene M and Ndiaye M. 2015. Production d'alevins de Tilapia (Oreochromis niloticus) avec 3 aliments à base de sous-produits agro-industriels au Nord du Sénégal. Int. J. Biol. Chem.
Sci., 9(5): 2598-2606. DOI: 10.4314/ijbcs.v9i5.29.

Tambi NE. 2001. Analysis of household attitudes toward the purchase of livestock products and fish in Cameroon. Agric Econ., 26(2): 135-147. DOI: 10.1111/j.1574-0862.2001.tb00059.x.

Teleu NE, Ngatchou A. 2006. Première évaluation $\mathrm{du}$ secteur avicole au Cameroun; structure et importance du secteur avicole commercial et familial pour une meilleure compréhension de l'enjeu aviaire. Rapport des consultants nationaux.

Tomedi E. 2015. Etat des lieux de la pisciculture au Cameroun. Hôtel Hilton à Yaoundé, l’influenza 31 mars 2015.

Tiogué CT, Bibou A, Kenfack A, Tchoumboué J. 2020. Caractéristiques Socio-économiques et techniques des élevages piscicoles du Département du Mbam et Inoubou. Int. J. Biol. Chem. Sci., 14(3): 983-1000. DOI: 10.4314/ijbcs. v14i3.26.

Yoboue KP, Aboua BRD, Berte S, Coulibaly JK, Ouattara NI, Kouamelan EP. 2018. Impacts des exploitations piscicoles en cages flottantes sur la structure des macroinvertébrés benthiques de la lagune Ebrié (Côte d'Ivoire). Int. J. Biol. Chem. Sci., 12(2): 769-780. DOI: 10.4314/ijbcs.v12i2.12. 